\title{
Associative retrieval processes in free recall
}

\author{
MICHAEL J. KAHANA \\ Brandeis University, Waltham, Massachusetts
}

\begin{abstract}
I present a new method for analyzing associative processes in free recall. While previous research has emphasized the prominence of semantic organization, the present method illustrates the importance of association by contiguity. This is done by examining conditional response probabilities in the output sequence. For a given item recalled, I examine the probability and latency that it follows an item from a nearby or distant input position. These conditional probabilities and latencies, plotted as a function of the lag between studied items, reveal several regularities about output order in free recall. First, subjects tend to recall items more often and more rapidly from adjacent input positions than from remote input positions. Second, subjects are about twice as likely to recall adjacent pairs in the forward than in the backward direction and are significantly faster in doing so. These effects are observed at all positions in the output sequence. The asymmetry effect is theoretically significant because, in cued recall, nearly symmetric retrieval is found at all serial positions (Kahana, 1995; Murdock, 1962). An attempt is made to fit the search of associative memory model (Raaijmakers \& Shiffrin, 1980, 1981) with and without symmetric interitem associations to these data. Other models of free recall are also discussed.
\end{abstract}

The finding that subjects, when told to recall a list of items "in any order," recall categorically or associatively related items in neighboring output positions has provided a foundation for much of the organizational theorizing on human memory. One might even argue that it was these findings which caused a shift from the study of paired associate learning and serial recall in the $1950 \mathrm{~s}$ and early 1960s to the study of free recall in the mid to late 1960s. Research on serial recall was long concerned with distinguishing theories of positional associations from chained associations (Harcum, 1975), whereas research on free recall focused on interitem similarity and context-to-item associations as the bases for retrieval (Shuell, 1969; Tulving, 1968).

What happened to the idea that items studied for free recall are related by virtue of their contiguity? Asch and Ebenholz (1962) found that during free recall only $18 \%$ of the total number of sequential responses matched adjacent sequences of list items in the forward order. They also found no significant difference in the overall number of forward and backward transitions (not necessarily adjacent). ${ }^{1}$ On the basis of these findings, they concluded that "the order in which items were produced in free recall (which registered the course of acquisition) did not correspond notably to the order of earlier experience" (Asch \& Ebenholz, 1962, p. 19).

This research was supported in part by an NIH Individual NRSA (1 F32 NS09559-01) to the author and the W. M. Keck Foundation. I am thankful to Ben Murdock for providing me with the raw data from his experiments and for helpful discussions of many of the ideas presented in this article. Correspondence concerning this article should be addressed to M. J. Kahana, Center for Complex Systems, Brandeis University, Waltham, MA 02254-9110 (e-mail: kahana@fechner.ccs. brandeis.edu).
It is unlikely that this one paper extinguished the belief in chained associations in free recall. Rather, it seems that the success of the organizational theorists in deriving important principles of memory from the free-recall task led to a total disregard for the role of chained associations in free recall. As Tulving (1968) notes, "It looks as if the conceptual analyses of free recall have been developed not just in isolation, but almost in defiance of the traditional S-R models of behavior."

Another factor contributing to the shift of interest away from an associationist approach to free recall was the theoretical entanglement of the free-recall method with models based on the temporal partitioning of memory into separate short- and long-term stores. With these models, the emphasis became one of structure over process, and the challenge to researchers was to explain the numerous dissociations between prerecency and recency portions of the serial position curve (e.g., Atkinson \& Shiffrin, 1968; Glanzer \& Cunitz, 1966).

In this paper, I present a new method for examining the role of pairwise associations in free recall. Before turning to the proposed method, I discuss the major methods for analyzing "primary organization" in single-trial free recall.

\section{Serial Position Curves and Mean Output Position Curves}

In free recall, the serial position curve is characterized by a small primacy effect, a large recency effect, and a generally flat middle region (usually called asymptote). Figure 1 shows the classic serial position data from one of the conditions reported in Murdock (1962). Output order effects have been summarized by plotting mean output position as a function of serial (input) position. 


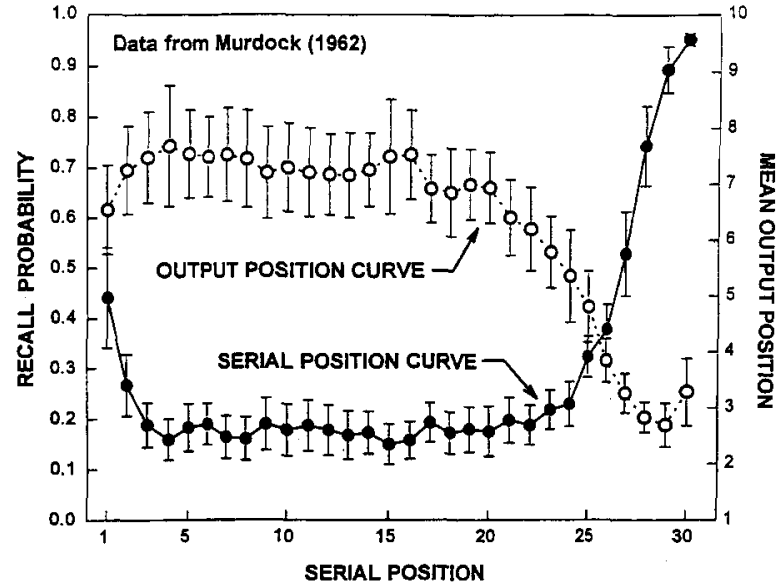

Figure 1. Serial position curve and mean output position curve in single-trial free recall. Data are from Murdock (1962). In this condition, 15 subjects studied 80 lists of 30 common words presented auditorily at a 1-sec rate. Error bars reflect $95 \%$ confidence intervals around each mean. The Loftus and Masson (1994, Appendix B) procedure for computing confidence intervals in within-subject designs was used.

The basic pattern is that items from the recency portion of the serial position curve are recalled first, while items from asymptote are recalled last. Furthermore, the items from the asymptotic portion of the serial position curve are at asymptote in the output position curve. The high correlation between the serial position curve and mean output position curve was noted by Deese and Kaufman (1957).

One interesting difference between mean output position curves and serial position curves is the "hook" at the end of the recency portion of the mean output position curve. For auditorily presented lists of words, recall begins about three items back, proceeds in forward order to the end of the list, and then moves to items in earlier list positions (Nilsson, Wright, \& Murdock, 1975). This pattern of recall produces the hook in the mean output position curve. The item that is recalled first (on average) is about two or three items from the end of the list. In contrast, with visually presented word lists, recall tends to begin with the last item and go backwards in a generally monotonic fashion.

\section{Conditional Response Probability and Latency Curves}

One of the central theoretical questions that comes up in the study of free recall is the nature of subject-generated retrieval cues. Neither serial position curves nor mean output position curves provide us with useful information about the specific item-by-item contingencies in output order. The equivalence of recall probability and output position for asymptotic items (often assumed to be retrieved from long-term memory) has suggested that there are no consistent relations between input order and output order for these items. Rather, it is generally as- sumed that retrieval of asymptotic items reflects a semantically and/or contextually guided search through longterm memory.

One way of interpreting the dynamics of retrieval in the asymptotic portion of the serial position curve is to examine conditional response probabilities. What is the likelihood of recalling item $x$ immediately followed by item $y$, conditional on recall of $y$ ? An even higher order statistic might measure the likelihood of recalling item $x$ followed by item $y$ followed by item $z$ conditional on recall of $z$.

As a means of characterizing these sequential contingencies in recall order, the probability of recalling item $y$ after item $x$, conditionalized on recall of item $y$, is plotted as a function of the lag $(x-y)$ for all items recalled after the third output position (see Figure 2). A lag of +1 implies that item $y=x+1$. Similarly, a lag of -1

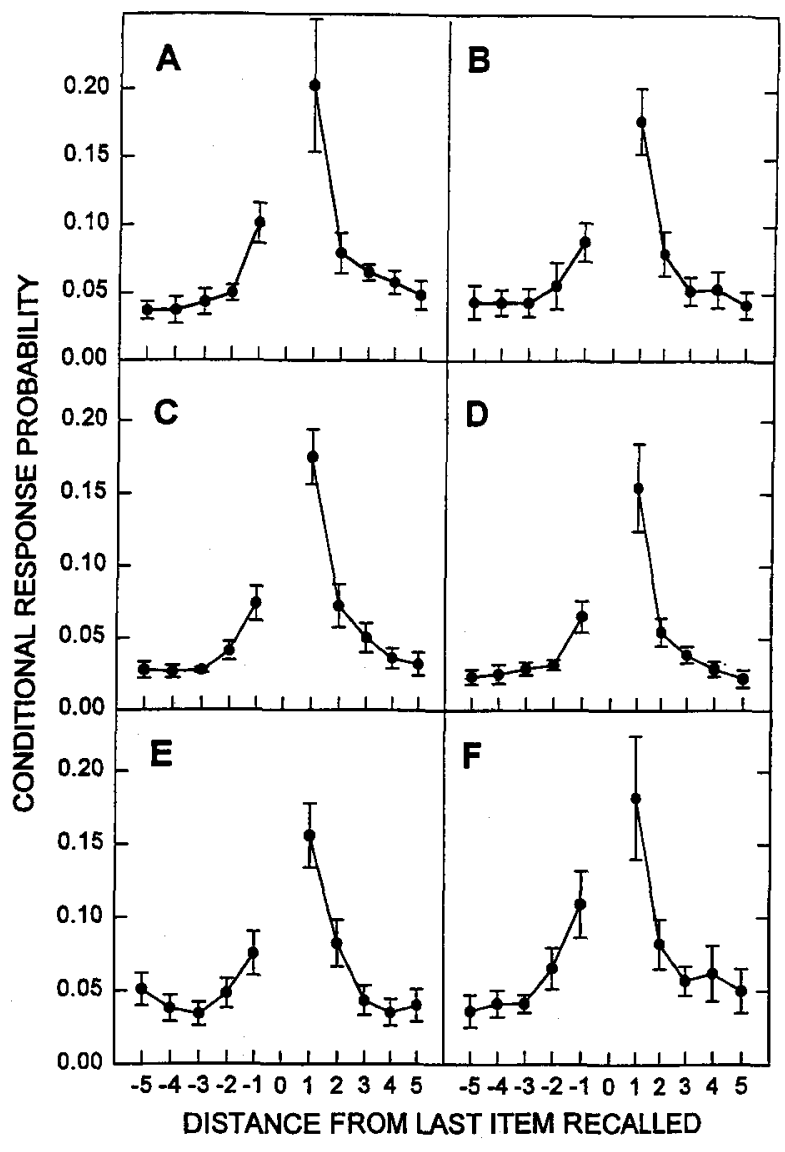

Figure 2. Conditional response probability curves for six studies of single-trial free recall. Panels A-D are based on data from Murdock (1962). Panel $E$ is based on data from Murdock and Okada (1970). Panel F is based on data from Murdock and Metcalfe (1978). (A) List length $=20$, auditory, $2 \mathrm{sec} /$ item. (B) List length $=20$, auditory, $1 \mathrm{sec} /$ item. (C) List length $=30$, auditory, $1 \mathrm{sec} /$ item. (D) List length $=40$, auditory, $1 \mathrm{sec} / \mathrm{item}$. (E) List length $=20$, visual, $1 \mathrm{sec} /$ item and $0.5 \mathrm{sec} /$ item combined. (F) List length $=20$, visual, $5 \mathrm{sec} /$ item. Error bars reflect $95 \%$ confidence intervals around each mean. The Loftus and Masson (1994, Appendix B) procedure for computing confidence intervals in within-subject designs was used. See text for details on computing conditional response probability curves. 
means that item $y=x-1$. As such, the absolute value of the lag is a measure of the degree of remoteness (at input) of successively recalled items. The sign of the lag indicates whether recall is in the forward (positive) or backward (negative) direction. Figure 2 shows conditional response probability curves based on free-recall data from six large free-recall experiments reported in three papers (Murdock, 1962; Murdock \& Metcalfe, 1978; Murdock \& Okada, 1970). ${ }^{2}$

Conditional response probability curves from all of these experiments show essentially identical patterns. When a given item is recalled, it tends to follow an item from a nearby input position and is twice as likely to follow the preceding adjacent item as it is the following adjacent item. In summary, items at contiguous input positions tend to be recalled together and in the forward order. This asymmetry is theoretically significant because cued recall yields equivalent forward and backward response probabilities across serial positions (Kahana, 1995; Murdock, 1962).

So far, these analyses have been based on data averaged across output positions (excluding the first three items recalled). A more detailed picture of recall order can be seen by looking at the conditional response probabilities at each position in the output sequence. Instead of examining transitions at each lag individually, I consider the average number of adjacent and remote, forward and backward transitions at each position in the output sequence. This analysis, applied to data from Murdock (1962; list length $=30$ ), is shown in Figure 3 .

It can be seen from these data that the advantage of forward-adjacent recalls is present at all output positions.

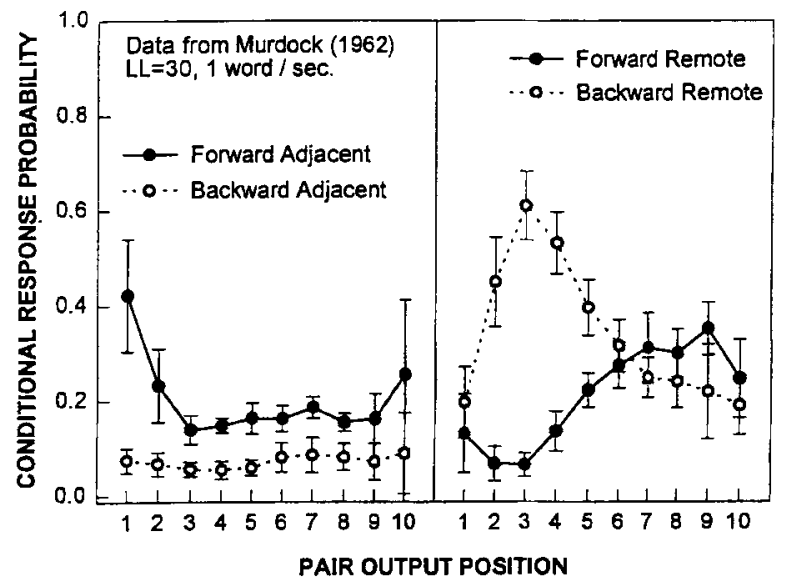

Figure 3. This figure shows adjacent $(\mathrm{lag}=1)$ and remote (sum of all lags $>3$ ) forward and backward conditional response probabilities at each pair output position (data from Murdock, 1962; list length $=30$ ). Pair output position $x$ refers to actual output positions $x$ and $x+1$. Consider an item from serial position $y$ recalled in output position $x$. A forward-adjacent recall refers to recalling item $y+1$ in output position $x+1$; a forward remote recall refers to the summed conditional probabilities of recalling items $y+4, y+5, \ldots$, $y+($ list length -1 ) in output position $x+1$. Error bars reflect $95 \%$ confidence intervals around each mean. The Loftus and Masson (1994, Appendix B) procedure for computing confidence intervals in within-subject designs was used.

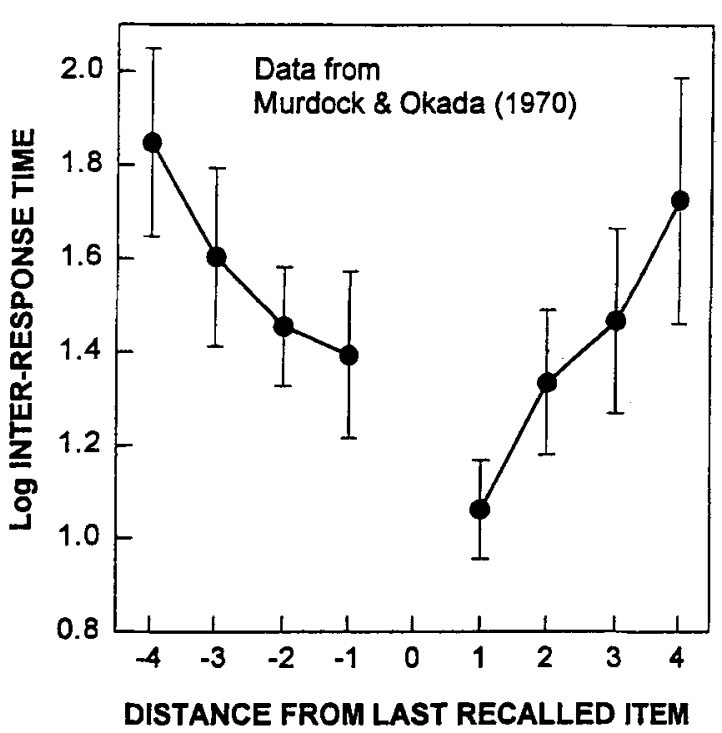

Figure 4. Conditional response-latency curve for data collapsed across both 1- and 2-sec presentation rates in Murdock and Okada (1970). Log interresponse times is computed as $\ln (1+$ IRT). Error bars reflect $95 \%$ confidence intervals around each mean. The Loftus and Masson (1994, Appendix B) procedure for computing confidence intervals in within-subject designs was used.

In early stages of recall, most transitions are forward adjacent. Later, forward and backward remote transitions are approximately of equal probability. It is unlikely that these late transitions are random; rather, they probably reflect semantic associations between list items.

Differences in strength are often associated with differences in response latency. Analyzing data on interresponse times collected by Murdock and Okada $(1970)^{3}$ allows us to ascertain whether asymmetry and adjacency effects observed in recall order are also observed in recall latency. Figure 4 shows the conditional response and latency curves for data collected by Murdock and Okada. The conditional response latency curve follows the same conventions as the conditional response probability curves described above. Log latencies were computed to reduce the effects of variability caused by long interresponse times. As shown in Figure 4, latencies were shortest when items from successive input positions were recalled in forward order. Latencies were somewhat longer for backward recalls. Both forward and backward recalls exhibit gradients as a function of lag.

\section{Summary of the Data}

Three basic findings emerge from these analyses. First, the probability of successive recall from adjacent input positions is about three times higher than the probability of successive recall from remote input positions. Second, the probability of adjacent forward recalls is about twice that of adjacent backward recalls. This forwardrecall advantage is present at all output positions. Third, 
adjacency and asymmetry effects are observed for latency as well as accuracy measures.

\section{Theoretical Analysis}

At a very general level, perhaps one can say that the conditional response probability and conditional response latency curves provide evidence for the role of contiguitybased associations in free recall. More specifically, however, it is useful to examine these curves in terms of the predictions of formal models of the free-recall task. Of all the models proposed to account for free recall, the search for associative memory (SAM) model (Gillund \& Shiffrin, 1984; Raaijmakers \& Shiffrin, 1980, 1981) presents the most comprehensive account of data from this task. What follows is a very brief summary of the SAM model. For a complete presentation, see Raaijmakers and Shiffrin (1980).

\section{The SAM Model of Free Recall}

The SAM model of free recall is an outgrowth of the Atkinson and Shiffrin (1968) buffer model. It consists of two storage systems: A limited-capacity short-term store (STS) and a long-term storage (LTS) associative network. Each new studied item enters STS. Once all of the registers in STS are full, a newly studied item replaces a randomly chosen item already in STS. According to SAM, items in STS are always available for immediate recall. A four-item rehearsal buffer will typically yield a good fit to the recency portion of the serial position curve.

LTS in SAM is modeled as an associative network. Items can be associated with each other, themselves, or context. For each unit of time that an item spends in STS, the strength of its association to context is incremented by parameter $a$ and its self-strength is incremented by parameter $c$. For each unit of time that two items spend together in STS, the strength of their association in LTS is incremented by parameter $b$. Items that are never together in STS have a residual interitem association given by parameter $d$. In Gillund and Shiffrin (1984), variability was introduced into the basic SAM model. To produce variability in the associative network, each associative strength is multiplied by a random variable drawn from a three-point distribution $(1,1-v$, or $1+v$, where $v$ is the variability parameter of the model).

Retrieval in SAM involves sampling memory with multiple cues. Initially, context is used as a cue to "sample" items in memory. If an item is "recovered," it is used, together with context, to sample another item from memory. An item cannot be recovered if the same retrieval cues failed to recover the item previously or if the item has already been recalled. Once there have been $L_{\max }$ consecutive failures at "recovery," retrieval is attempted with context alone. The overall process repeats itself until there are $K_{\max }$ "recovery" failures to any cues. Whenever an item is recovered, its self-strength is incremented by parameter $g$, its association to context is incremented by parameter $e$, and if another item was one of the retrieval cues, the interitem associative strength is incremented by parameter $f$. The equations for sampling and recovery probabilities are given in Raaijmakers and Shiffrin (1980).

SAM differs from other contemporary memory models in that it does not make explicit assumptions about the representation of items in memory or the associative mechanism. Rather, SAM maintains an abstract characterization of the probability of successful retrieval given a set of retrieval cues.

Because items in nearby input positions spend more time together in the rehearsal buffer, conditional response probabilities should be greater for adjacent items than for remote items. However, because interitem associations in SAM are bidirectional, the observed asymmetry in forward and backward recall may not be predicted.

\section{Simulations}

To fully examine the SAM model's ability to account for the free-recall data, the model was simultaneously fit to the serial position curve, mean output position curve, and conditional response probability data from the $30-$ item/1-sec condition in Murdock (1962). In fitting SAM to these data, three parameters were fixed: The size of the rehearsal buffer, $r$, was set at 4 and the $K_{\max }$ and $L_{\max }$ parameters were set at 30 and 3, respectively. Simplex (Nelder \& Mead, 1965) was used to estimate the remaining 8 parameters that minimized the chi-square statistic. For each simplex iteration, results were averaged over 10,000 replications of the SAM model.

The best-fitting parameters were: $a=0.0204, b=$ $0.5666, c=0.6394, d=0.4376, e=0.546, f=0.5827$, $g=0.5674$, and $v=0.1783$. The fit of the model to the data is depicted in Figure 5. The fits of the serial position $\left[\chi^{2}(22)=168.7\right]$ and output position $\left[\chi^{2}(22)=122.2\right]$ curves were reasonable. In contrast, the model was largely unsuccessful in fitting the conditional response probabilities $\left[\chi^{2}(32)=1,169\right]$. To see if these results were stable, I repeated the simulation for different values of the fixed parameters. Setting the size of the rehearsal buffer to 3 instead of 4 increased the chi-square values considerably, and trying several different values of $K_{\max }$ and $L_{\max }$ also did not improve the fit.

As can be seen in Figure 5, the SAM model fails to account for the difference between forward- and backwardadjacent recalls at any of the output positions. At early output positions, SAM far overpredicts the probability of forward remote recalls. Finally, SAM predicts an abrupt transition in remote recalls after the fourth output position, whereas the data show a gradual transition.

Why does SAM make these predictions, and how could it be fixed to handle the data? First, SAM assumes symmetric associations between items in memory. This may account for SAM's inability to produce the asymmetry in forward and backward recall. Second, because retrieval in SAM reflects output from the short-term store followed by search of associative memory, there is a sudden shift 

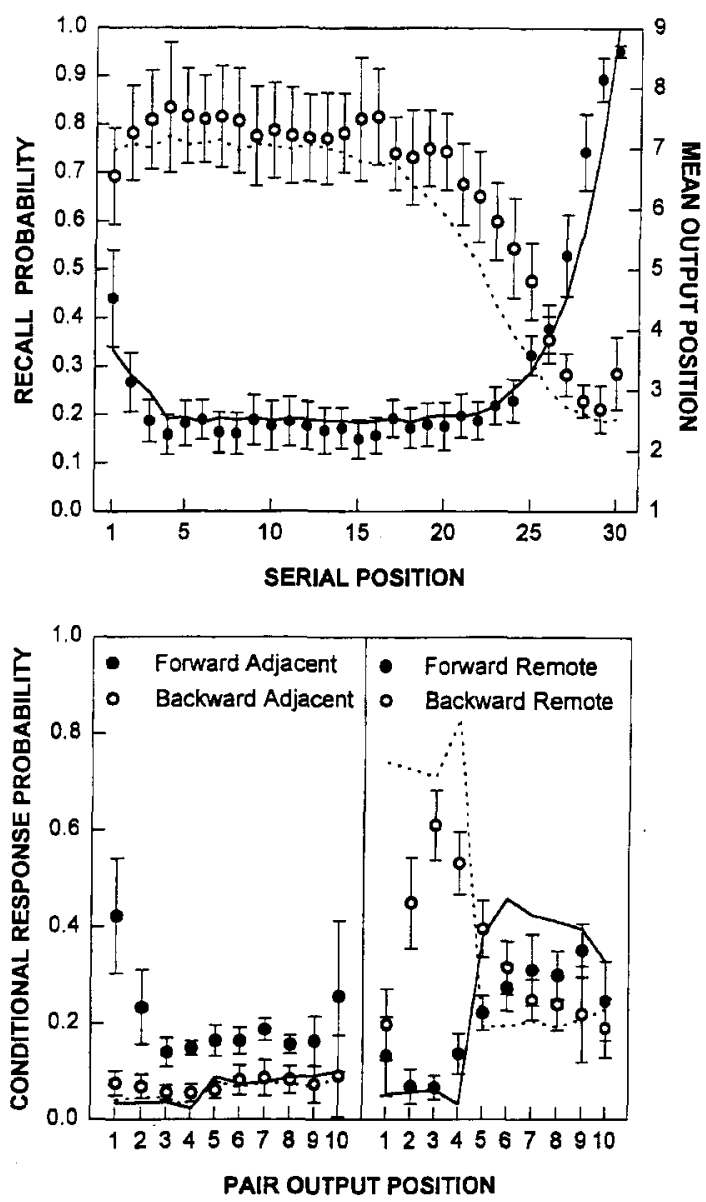

Figure 5. This figure shows the best fit of the SAM model of free recall (Raaijmakers \& Shiffrin, 1980) to the serial position data and mean output position data (top panel) and the conditional response probability data (bottom panel) from the 30-1 condition in Murdock (1962). Error bars reflect $95 \%$ confidence intervals around each mean. The Loftus and Masson (1994, Appendix B) procedure for computing confidence intervals in within-subject designs was used.

in strategy at the fourth output position. This problem might be solved if the size of the rehearsal buffer were varied across simulated trials.

In an effort to bring SAM's predictions closer to the data, four modifications were introduced. First, separate parameters for forward and backward interitem associations were added (both during study and test). Second, the rehearsal buffer size, $r$, was randomly set to $2,3,4$, 5 , or 6 on each trial (with probabilities of $0.1, .22, .36$, .22 , and .10, respectively). Third, the rehearsal rule employed by Phillips, Shiffrin, and Atkinson (1967) was adopted. According to that rule, the probability of the $i$ th buffer item being displaced was calculated as:

$$
d(i)=\frac{\delta(1-\delta)^{i-1}}{1-(1-\delta)^{r}}
$$

where $\delta$ is a new free parameter of the model. Using this rehearsal scheme, the older the item in the rehearsal buffer, the more likely it will be displaced. This scheme results in more rehearsals for recently studied items than for remote items. Taken alone, this change in SAM greatly improved the fit to the conditional response probability data, but also wiped out the primacy effect. To recover the primacy effect, an attentional weighting factor was added to the rehearsal buffer. This factor was used in Gillund and Shiffrin (1984), but not in Raaijmakers and Shiffrin (1981). Finally, with the primacy effect back, the fit of the revised model is as shown in Figure 6 . The best-fitting parameters were: $a=0.082, b 1=$ 0.626 (forward), $b 2=0.420$ (backward), $c=0.777, d=$ $0.075, e=0.401, f 1=0.461$ (forward), $f 2=0.201$ (backward), $g=0.619, v=0.197, \delta=0.423$.

A major improvement can be seen in the model's fit to the conditional response probability data. Although the fit is not numerically spectacular, the revised model actually provides a reasonable account of the retrieval process $\left[\chi^{2}(29)=251.25\right]$. The revised model's fit to the
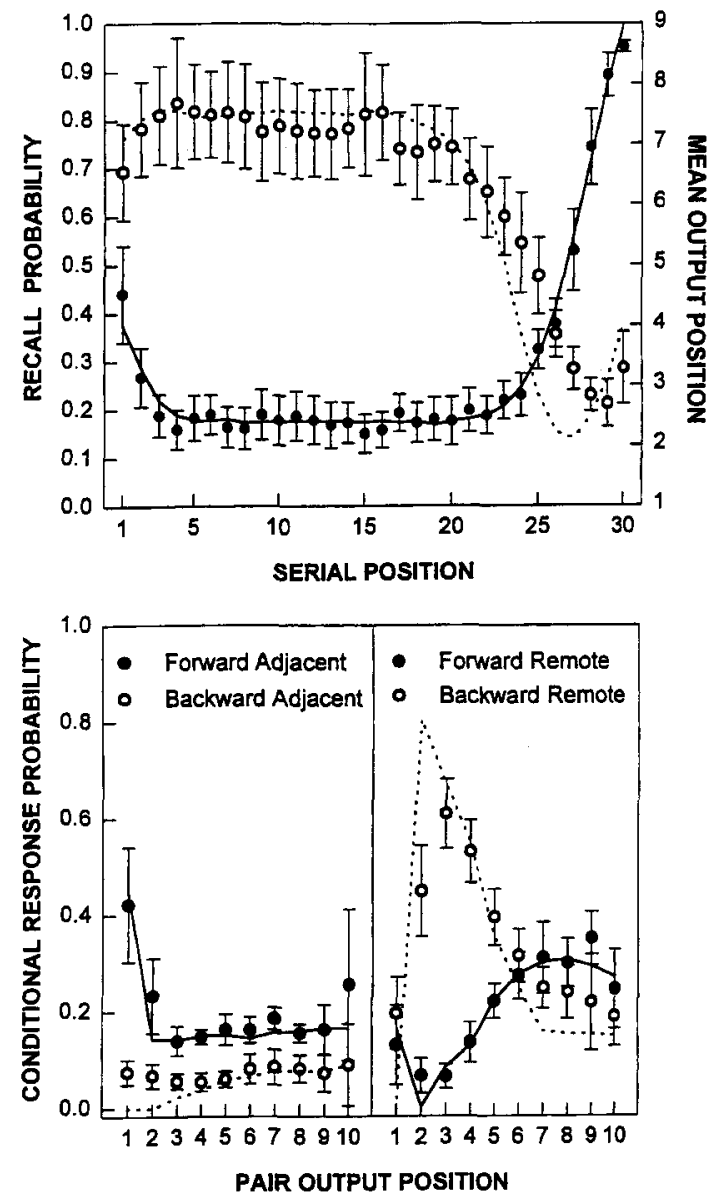

Figure 6. 'This figure shows the best fit of a modified version of the SAM free-recall model to the serial position data and mean output position data (top panel) and the conditional response probability data (bottom panel) from the 30-1 condition in Murdock (1962). Error bars reflect $95 \%$ confidence intervals around each mean. The Loftus and Masson (1994, Appendix B) procedure for computing confidence intervals in within-subject designs was used. 
serial position curve $\left[\chi^{2}(19)=85.0\right]$ and output position curve $\left[\chi^{2}(19)=199.8\right]$ did not change substantially (with the exception of the exaggerated hook now present in the output position curve).

\section{Conclusions}

This paper presents a quantitative method for examining the role of interitem associations in free recall. Plotting the probability of recalling an item from serial position $x$ followed by an item from serial position $y$ for different lags reveals strong adjacency effects, a forward-recall advantage, and shallow gradients beyond lag 2 . The tendency to recall adjacent pairs in the forward order is highly pronounced for the first few output positions and then settles to a level of about twice the backward-recall probability for the rest of the recall process.

The SAM model provides a reasonable fit to both serial position and mean output position curves. However, in its original version, the model does not adequately account for the conditional response probability data. The original model assumes symmetric increments of forward and backward interitem associations. To bring the model within striking distance of the data, three basic modifications were needed: (1) separate parameters for forward and backward interitem associations, (2) a variable buffer size, and (3) a rehearsal rule that prefers nearby items (Phillips et al., 1967).

Another successful model of single-trial free recall is Metcalfe and Murdock's (1981) chaining model. The chaining model belongs to a class of distributed memory models that use convolution and correlation as the associative storage and retrieval mechanisms. Because convolution is commutative, forward and backward associations are of equal strength. Metcalfe and Murdock suggest that recall begins by cuing memory with the lastrehearsed item. Chaining through memory continues until a specified number of successive retrieval failures. Subsequently, retrieval is reinitiated with a beginningof-list cue (associated with the first studied item). Forward chaining continues until another series of retrieval failures. Because of this two-stage retrieval process, conditional response probabilities should exhibit a backwardrecall advantage for early output positions, and a forwardrecall advantage for late output positions. It is clear from Figure 3 that this is not the case. Adjacent forward recalls are higher than adjacent backward recalls at all output positions, but especially at early output positions. Subjects' tendency to move backward at early stages of output and forward at later stages of output is seen only in remote recalls.

In addition to the SAM buffer model (Raaijmakers \& Shiffrin, 1981) and the associative chaining model (Metcalfe \& Murdock, 1981), two other approaches to studying free recall deserve attention. In an effort to account for the ubiquity of exponential interresponse time functions in free recall, Rohrer and Wixted (1994) reintroduced the traditional notion of random search without replacement. The analyses presented in this paper demonstrate that free recall of items from asymptotic serial positions simultaneously exhibit exponential growth in interresponse times and conditional response latency effects (see Figure 4 and Murdock \& Okada, 1970). A random-search model cannot account for conditional response latency effects (or the effects of semantic clustering reported elsewhere in the literature). Despite these limitations, Rohrer and Wixted's approach may still be useful. The fundamental question they raise, which is not addressed by the present data, is whether the increase in response latency with output position is a consequence of the resampling of items already recalled or some other process.

Glenberg and Swanson's (1986) temporal distinctiveness theory (TDT) has been developed to account for free recall in the continuous distractor paradigm. In this paradigm, direct interitem associations are either weakened or eliminated by the distractor task and the focus is on temporal retrieval strategies. According to TDT, items are sampled from temporally defined search sets in memory. These search sets are assumed to be narrow for recent items but broad for prior list items. In the absence of strong interitem associations, this type of model predicts symmetric gradients in the conditional response probability curves. The gradients should be narrow early in recall and broaden with output position. Although TDT makes testable predictions about conditional response probability functions in free recall, these predictions cannot be tested using data from the standard version of the task.

\section{Summary}

Previous research on free recall has focused on semantic relations between list items (e.g., Brown, Conover, Flores, \& Goodman, 1991; Cooke, Durso, \& Schvaneveldt, 1986; Romney, Brewer, \& Batchelder, 1993; see Shuell, 1969, for a review of the earlier literature) to the exclusion of association by contiguity. Some potential reasons for this were discussed in the introduction. Conditional response probability and latency analyses of a number of large free-recall studies suggest that the neglect of contiguity-based associative processes in free recall is unwarranted. In order to predict free-recall performance, both contiguity-based associative processes and semantic relations among list items need to be considered.

\section{REFERENCES}

Asch, S. E., \& Ebenholtz, S. M. (1962). The process of free recall: Evidence for non-associative factors in acquisition and retention. Journal of Experimental Psychology, 54, 3-31.

Atkinson, R. C., \& Shiffrin, R. M. (1968). Human memory: A proposed system and its control processes. In K. W. Spence \& J. T. Spence (Eds.), The psychology of learning and motivation: $A d-$ vances in research and theory (Vol. 2, pp. 89-195). New York: Academic Press.

Brown, S. C., Conover, J. N., Flores, L. M., \& Goodman, K. M. (1991). Clustering and recall: Do high clusterers recall more than 
low clusterers because of clustering? Journal of Experimental Psychology: Learning, Memory, \& Cognition, 17, 710-721.

Cooke, N. M., Durso, F. T., \& Schvaneveldt, R. W. (1986). Recall and measures of memory organization. Journal of Experimental Psychology: Learning, Memory, \& Cognition, 12, 538-549.

DeEse, J., \& Kaufman, R. A. (1957). Serial effects in recall of unorganized and sequentially organized verbal material. Journal of Experimental Psychology, 54, 180-187.

Gillund, G., \& Shiffrin, R. M. (1984). A retrieval model for both recognition and recall. Psychological Review, 91, 1-67.

GlanZer, M., \& CUNITZ, A. R. (1966). Two storage mechanisms in free recall. Journal of Verbal Learning \& Verbal Behavior, 5, 351360

Glenberg, A. M., \& Swanson, N. G. (1986). A temporal distinctiveness theory of recency and modality effects. Journal of Experimental Psychology: Learning, Memory, \& Cognition, 12, 3-15.

Harcum, E. R. (1975). Serial learning and paralearning. New York: Wiley.

Kahana, M. J. (1995). Associative symmetry and memory theory. Manuscript submitted for publication

LofTUS, G. R., \& Masson, M. E. J. (1994). Using confidence intervals in within-subject designs. Psychonomic Bulletin \& Review, 1, 476490.

Metcalfe, J. A., \& Murdock, B. B. (1981). An encoding and retrieval model of single-trial free recall. Journal of Verbal Learning \& Verbal Behavior, 20, 161-189.

MURDOCK, B. B. (1962). The serial position effect of free recall. Journal of Experimental Psychology, 64, 482-488.

Murdock, B. B., \& Metcal.fe, J. A. (1978). Controlled rehearsal in single-trial free recall. Journal of Verbal Learning \& Verbal Behavior, 17, 309-324.

Murdock, B. B., \& OKadA, R. (1970). Interresponse times in singletrial free recall. Journal of Verbal Learning \& Verbal Behavior, $\mathbf{8 6}$, 263-267.

Nairne, J. S., Riegler, G. L., \& Serra, M. (1991). Dissociative effects of generation on item and order retention. Journal of Experimental Psychology: Learning, Memory, \& Cognition, 17, 702-709.

Nelder, J. A., \& MEAD, R. (1965). A simplex method for function minimization. Computer Journal, 7, 308-313.

Nilsson, L.-G., Wright, E., \& Murdock, B. B. (1975). The effects of visual presentation method on single-trial free recall. Memory \& Cog nition, 3, 427-433.

Phillips, J. L., Shiffrin, R. J., \& Atkinson, R. C. (1967). The effects of list length on short-term memory. Journal of Verbal Learning \& Verbal Behavior, 6, 303-311.

RAAIJMAKERS, J. G. W., \& Shiffrin, R. M. (1980). SAM: A theory of probabilistic search of associative memory. In G. H. Bower (Ed.), The psychology of learning and motivation: Advances in research and theory (Vol. 14, pp. 207-262). New York: Academic Press.

RaAiJmakers, J. G. W., \& Shiffrin, R. M. (1981). Search of associative memory. Psychological Review, 88, 93-134.

ROHRER, D., \& WIXTED, J. T. (1994). An analysis of latency and interresponse time in free recall. Memory \& Cognition, 22, 511-524.

Romney, A. K., Brewer, D. D., \& Batchelder, W. H. (1993). Predicting clustering from semantic structure. Psychological Science, 4, 28-34.

SHUELL, T. J. (1969). Clustering and organization in free recall. Psychological Bulletin, 72, 353-374

TulviNG, E. (1968). Theoretical issues in free recall. In T. R. Dixon \& D. L. Horton (Eds.), Verbal behavior and general behavior theory (pp. 2-36). Englewood Cliffs, NJ: Prentice-Hall.

\section{NOTES}

1. In a more recent study, Nairne, Riegler, and Serra (1991) found some evidence for forward seriation in the free-recall of eight-item lists. The percentage of adjacent recalls in the forward direction was 0.68 , reliably above the chance level of 0.50

2 . To get reliable data on conditional response probabilities, one needs fairly large data sets. Murdock (1962) is probably the largest freerecall study ever conducted with practiced subjects. Data for each condition are averaged from over 1,200 free-recall trials. In Murdock and Okada (1970), there are 720 trials for each of the presentation-rate conditions (slow $=1 \mathrm{item} / \mathrm{sec}$, fast $=2$ items $/ \mathrm{sec}$ ). Because of the low recall levels in this study, data from the two presentation-rate conditions were combined for the conditional analyses. In Murdock and Metcalfe (1978), there are 512 lists in the overt rehearsal condition.

3. To determine interresponse times, Murdock and Okada (1970) tested subjects individually in soundproof rooms where the entire test session was tape-recorded. The recorded protocols were analyzed using a sound meter and a plotter to measure the onset-onset interresponse times for all of the recalled words.

(Manuscript received December 21, 1993; revision accepted for publication March 13, 1995.) 